\title{
The ELISe electron rare isotope scattering experiment - status and perspectives
}

\author{
Haik Simon ${ }^{1}$ \\ GSI Helmholtzzentrum für Schwerionenforschung $\mathrm{GmbH}$ \\ Planckstrasse 1, 64291 Darmstadt, Germany \\ E-mail: h.simon@gsi.de
}

\section{for the ELISe collaboration}

http://www.gsi.de/forschung/fair_experiments/elise/collaboration.html

In this paper ELISe's core experimental programme to be carried out at the New Experimental Storage Ring (NESR) will be briefly outlined and used to motivate the design parameters for the installations. The main technical developments heading towards the preparation of technical design reports for this very challenging electron RIB scattering experiment using two intersecting storage rings and a high resolution magnetic spectrometer will be described in this contribution.

8th International Conference on Nuclear Physics at Storage Rings (Stori11)

Laboratori Nazionali di Frascati dell'INFN , Italy

October 9-14, 201

\footnotetext{
${ }^{1}$ Speaker
} 


\section{Introduction}

The prospected high intensities for the FAIR radioactive beam facility [1] will allow for the first time to intersect two storage rings, one for circulating rare ion beams (RIBs) called New Experimental Storage Ring (NESR) and a small electron storage ring (EAR), that could be also filled with antiprotons for the AIC experiment, in order to perform colliding beam experiments with sufficient luminosity. The main ingredients for this intensity increase are an enhanced injector-synchrotron chain with increased intensities and shorter repetition rates, providing one to two orders of magnitude more beam compared to GSI's previous UNILACSIS accelerator performance around the year 2000 when the first planning steps for FAIR have been initiated, and -likewise important- the newly designed Super-FRS [2] fragment separator. This magnetic separator consists of a two stage pre-separator, coping with the very high beam intensities, and a main separator with four stages for each of the three attached branches: a high energy branch (HEB) hosting the $\mathrm{R}^{3} \mathrm{~B}$ experiment, a low energy branch (LEB) to be used for nuclear spectroscopy (HI/DESPEC), laser spectroscopy (LASPEC), and ion trap (MATS) experiments and the ring branch (comprising the NESR storage ring) with its experimental program including ELISe's being described elsewhere in these proceedings. The separator will allow selecting interesting species with almost no transmission losses from fragmentation reactions reaching very far from stability as well as for fission fragments, both with large demands on the achievable acceptance figures.

Introducing the electron as novel purely leptonic probe into the field of RIB physics is attractive as it will allow overcoming unavoidable shortcomings of the well adopted methods being typically used for their study. As example one may take coulomb excitation in inverse kinematics using highly charged targets as a source of virtual photons. This method is generally accepted in order to study the electromagnetic response of RIBs and is especially very suited for low intense beams due to its high sensitivity. However, the excitation energy put into the radioactive species has to be reconstructed from all decay products including gamma rays as the excitation process is in general not observable. This introduces problems when being forced to detect many reaction products in coincidence and suffers in addition from unavoidable background distributions from hadronic processes introduced through the target. In electron scattering studies the excitation energy could be directly measured by detecting the recoiling electron in a magnet spectrometer. One may in addition expect to be able to reconstruct the multipolarities of the excitation by observing the typical angular distributions for the electrons. Excitation and de-excitation process could be studied separately as in colliding beam kinematics both recoil electron are leaving the interaction zone and can thus be measured.

Electron scattering on RIBs is only elsewhere foreseen [3] and currently being set up at the RIKEN institute in Japan. Here the ions from an ISOL source will be trapped in the electrostatic potential of a circulating stored electron beam with the help of additional electrodes surrounding the interaction region. This so-called SCRIT method [4] is especially suitable for ISOL beams and will allow performing experiments in fixed-target kinematics. 


\section{Physics program and requirements for the experimental setup of ELISe}

The physics program of the ELISe is being discussed in more detail by KalantarNayestanaki et al. elsewhere in these proceedings, in references [5,6], and can be briefly summarized as follows. We want to:

(a) Obtain charge distributions for exotic nuclei from elastic electron scattering.

(b) Get access via inelastic electron scattering to low-lying collective states, giant resonances, and (new) collective (soft) modes with a high selectivity to different multipolarities.

(c) Study electro fission processes likewise, with a simultaneous detection of all fission fragments.

(d) Study direct reactions induced by quasi-free scattering such as (e,e.N), and cluster knockout providing information on the internal nuclear structure and nucleon nucleon correlations.

This list reflects the opportunities arising from the advantages using the point-like, purely electromagnetic probe which in turn implies that the interaction is weak and cross sections are small. This is advantageous as practically all interactions can be treated as single-step processes. However, limitations will arise from the achievable luminosity for these interactions that are mainly governed by: (i) beam production rates and transport losses, (ii) decay losses by nuclear and atomic life-time while cooling down the beam in order to achieve good beam quality, starting from the bad emittance from the violent production process, and (iii) the achievable small focus at the interaction region.

\begin{tabular}{|c|c|c|c|}
\hline Reaction & Deduced Quantity & RIB & $\begin{array}{l}\text { Luminosity } \\
\left(\mathrm{cm}^{-2} \mathrm{~s}^{-1}\right)\end{array}$ \\
\hline $\begin{array}{l}\text { Elastic scattering } \\
\text { at small } q\end{array}$ & r.m.s. charge radii & $\begin{array}{l}\text { Light } \\
\text { Medium }\end{array}$ & $10^{24}$ \\
\hline $\begin{array}{l}\text { First minimum in } \\
\text { elastic form factor }\end{array}$ & $\begin{array}{l}\text { Density distribution with } \\
2 \text { parameters }\end{array}$ & $\begin{array}{l}\text { Light } \\
\text { Medium } \\
\text { Heavy }\end{array}$ & $\begin{array}{l}10^{28} \\
10^{26} \\
10^{24}\end{array}$ \\
\hline $\begin{array}{l}\text { Second minimum in } \\
\text { elastic form factor }\end{array}$ & $\begin{array}{l}\text { Density distribution with } \\
3 \text { parameters }\end{array}$ & $\begin{array}{l}\text { Medium } \\
\text { Heavy }\end{array}$ & $\begin{array}{l}10^{29} \\
10^{26}\end{array}$ \\
\hline Giant resonances & Energy, width, strength, decays & $\begin{array}{l}\text { Medium } \\
\text { Heavy }\end{array}$ & $\begin{array}{l}10^{28} \\
10^{28}\end{array}$ \\
\hline $\begin{array}{l}\text { Quasi-elastic } \\
\text { scattering }\end{array}$ & $\begin{array}{l}\text { Spectroscopic factors, spectral } \\
\text { function, momentum distributions }\end{array}$ & Light & $10^{29}$ \\
\hline
\end{tabular}

Table1: Required luminosities for various studies. The given values are based on rate estimates for a maximally four week beam time. 


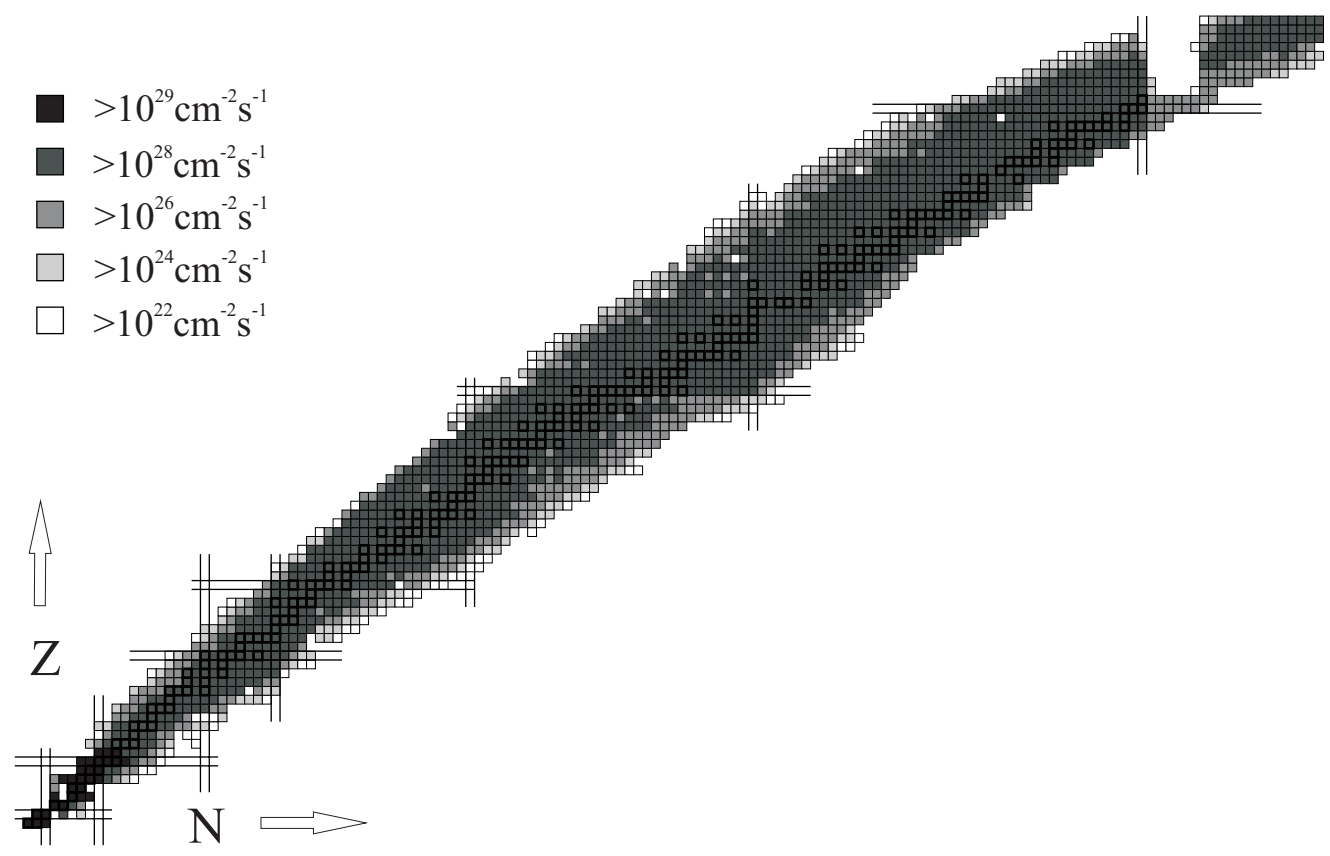

Figure 1: Achievable Luminosities in the ELISe experiment. For details, see text.

In Table 1 the necessary luminosities for different reaction types are given. It becomes clear, that the rates are moderate compared to contemporary stable beam physics experiments being owed to the fact, that the required momentum and energy transfer for the studies in question is achieved at small scattering angles of a few degrees in the centre-of-mass system. These correspond in turn to rather large Mott cross sections for the processes. In Figure 1 the result of a simulation for the here-presented accelerator and spectrometer setup is shown, taking into account production yields and separation efficiencies in conjunction with all abovementioned loss processes in the Super-FRS and the CR-RESR-NESR storage ring complex. It becomes clear, that all requirements are met and a viable experimental program could be carried out, once the experiment - being part of the FAIR core facility - would be build.

Another important issue is the achievable resolution of the electron spectrometer system. Colliding beam kinematics leads to a potential reduction of excitation energy resolution in the moving target like ion. Given the planned collider parameters, electron beam energies ranging from 125 up to $500 \mathrm{MeV}$ and ion energies ranging from about $100 \mathrm{MeV}$ per nucleon up to $740 \mathrm{MeV}$ per nucleon for NESR, the excitation resolution becomes scattering angle dependent. The centre-of-mass energy for the electrons ranges up to $1.5 \mathrm{GeV}$ in this case. In Figure 2 the effect is exemplified by a simulation where a colliding beam kinematics has been assumed for a medium heavy nucleus. It can be seen that for a given angular and momentum resolution, and for a chosen solid angle with maximum rate, the reconstructed excitation energy in the target like projectile is worse compared to a fixed-target experiment with the same electron spectrometer properties. By subsequently reducing the beams energy at the expense of achievable luminosity, the resolution can be gained back. It becomes clear, by looking at the right-hand side panels, that the electron spectrometer parameters in our design are determined by the physics case. In the following sections, both the in-ring spectrometer design together 
with the interaction zone in the EAR will be presented. Their design will eventually determine the overall geometry of the ELISe experiment.

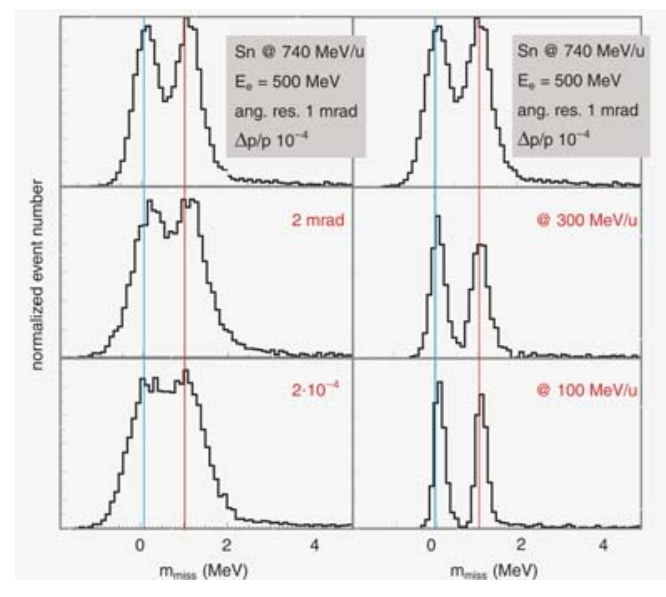

Figure 2:

Achievable resolution in colliding beam kinematics, assuming a medium heavy (A=132, Z=50) RIB with an artificial excited state at $\mathrm{E}^{*}=1 \mathrm{MeV}$. An electron spectrometer with a momentum resolution of $\delta \mathrm{p} / \mathrm{p}=10^{-4}$ and 1 mrad angular resolution is chosen.

Left panels: (top) Achievable resolution at $740 \mathrm{MeV} / \mathrm{u}$ beam energy, (middle, bottom) effect of worsening angular and energy resolution, respectively. Right panels: (top to bottom) gaining back resolution by reducing the ion energy at the expense of achievable luminosity.

\section{The EAR ring}

The Electron Antiproton Ring (EAR) in the bypass section of the NESR [6] is shown in Figure 3. The bypass is used to separate the ultra-high vacuum of the NESR ( $\sim 10^{-11} \mathrm{mbar}$ ) from the comparatively bad vacuum in the electron storage ring, being affected by the desorption caused by the radiative cooling of the electron beam. It provides additional space for a dedicated bunching system for the collider mode (right-hand side) and for experimental installations for fragment detection (left-hand side). Beam-beam forces and space charge effects have been considered carefully to come up with the maximal bunch populations in the rings. These are taken into account in the simulation presented earlier and allow keeping the beam emittances at bearable levels.

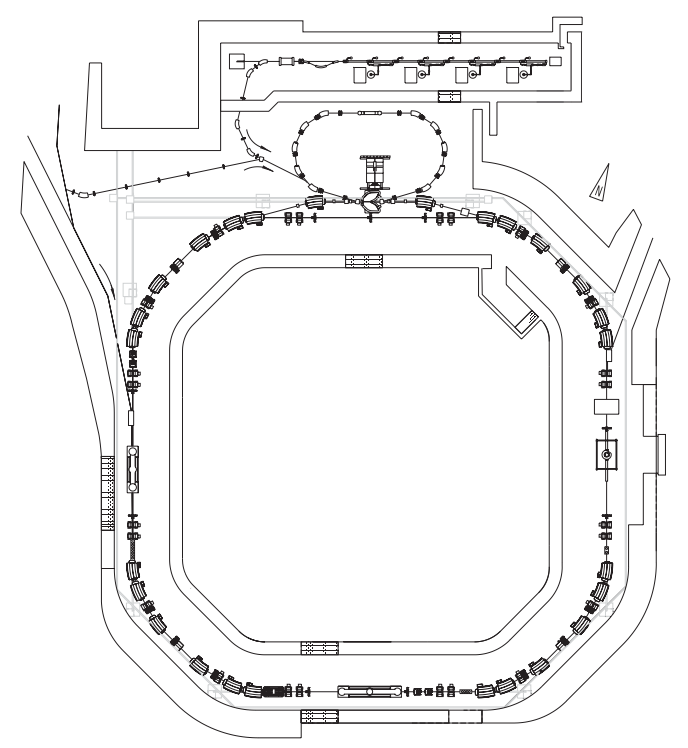

Figure 3:

Layout of NESR (circumference $222.916 \mathrm{~m}$ ) at the bottom and EAR $(53.693 \mathrm{~m})$ at the top. RIBs and antiprotons enter via the beam lines shown in the top left corner. Antiprotons can be guided with a special transfer line into the EAR The LINAC topmost allows to fill the EAR with electrons. The bypass region equipped with the LAHReS electron spectrometer are described in the text. 


\subsection{The interaction zone}

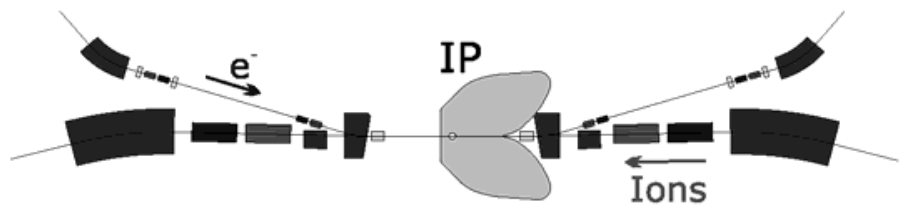

\section{Figure 4:}

Layout of the bypass section [6] at the interaction region (IP).

Particular efforts have been put in the design of the interaction region where several challenging demands have to be met. First, the arrangement should allow focusing the beams to minimal transverse sizes $\left(\sigma_{x, y}=220 \mu \mathrm{m}, 85 \mu \mathrm{m}\right)$ of the overlap zone, as the luminosity $L \propto 1 / \sigma_{\mathrm{x}} \sigma_{\mathrm{y}}$ is strongly dependent on them. Second, the section should provide enough ion-optical flexibility in order to steer the beams while optimizing their relative angles and positions. Third, the magnetic elements to the left-hand side of the interaction region act also as elements of a tracking spectrometer for target-like fragments, so that a cone of $\pm 20 \mathrm{mrad}$ is to be kept free from material. The simulation calculations of the dynamic apertures for both NESR and EAR in collider mode show satisfactory results. Due to the delay of the realization of the ring experiments the recently developed fragment spectrometer components will be first used in a $\mathrm{R}^{3} \mathrm{~B} /$ Cave-C beam campaign called SOFIA aiming for studying fission processes with simultaneous detection of both fission fragments.

\section{The LAHReS spectrometer}

The heart of the ELISe experiment is the Large Acceptance High Resolution electron Spectrometer shown in Figure 3. The current design foresees a pre-deflector keeping the beam region field free by the design of the magnet and shielding components. The pre-deflector acts as a dipole deflecting the beam horizontally into a QHD spectrometer deflecting the beam in vertical direction.

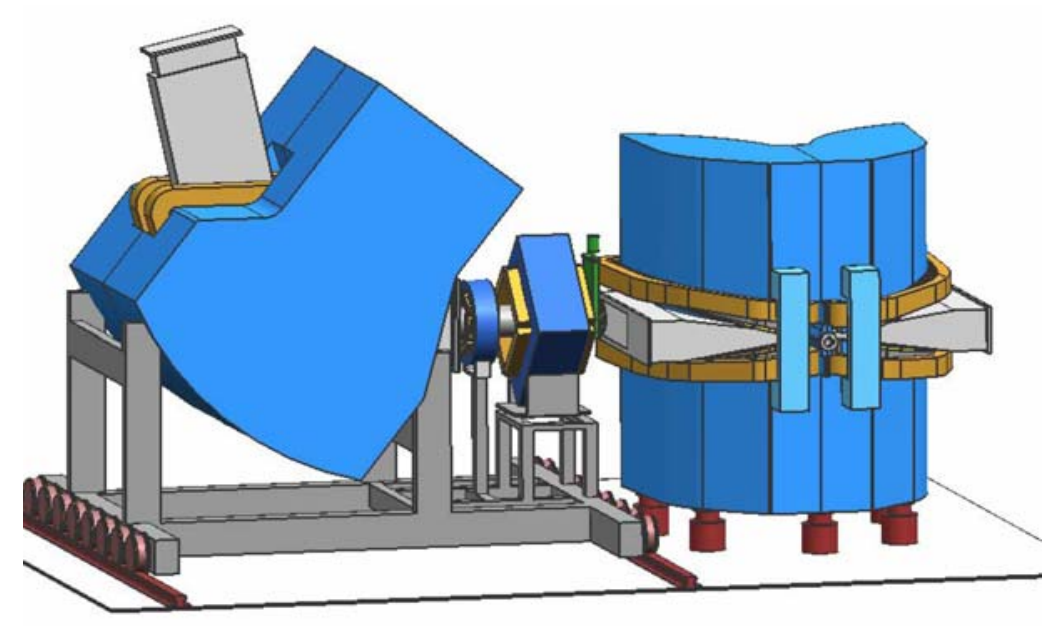

Figure 3:

Overview of the The Large Acceptance High Resolution $\mathrm{D}_{\text {hor }} \mathrm{QHD}_{\text {vert }}{ }^{-}$ electron Spectrometer [7]. The intersecting beams traverse the predeflector $\left(D_{\text {hor }}\right)$ at the right through the magnetic shield pipe in its middle. 
Ion optical simulation calculations using the COSY INFINITY code yield a momentum resolution that is twice better than required a angular resolution of $1 \mathrm{mrad}$ and a solid angle coverage of $12.9 \mathrm{msr}$ at $19^{\circ}$ deflection angle in the laboratory. However, for the lowest achievable scattering angle of about $11.4^{\circ}$ which corresponds to a momentum transfer of e.g. $q=0.1 \mathrm{GeV} / \mathrm{c}$ at $500 \mathrm{MeV}$ electron energy, or respectively smaller if the electron energy is further reduced, over-focusing appears which leads to a dramatic decrease in covered solid angle at small scattering angles. This is in particular unsatisfactory, as at the smallest angles exploratory charge radii studies on an absolute scale could be performed using short lived RIBs with life times in the order of a few $100 \mathrm{~ms}$.

\subsection{Further Improvements}

The situation can be cured by replacing the clam-shell design of the pre-deflector with a constant gap as shown in Figure 4. The simplified design keeping the same good resolution bears several advantages: (i) the over-focussing for the lowest angles is avoided, leading to a five-fold increase in solid angle coverage for the smallest scattering angles. The coverage at larger angles is only very little lowered. (ii) A homogeneous field in a constant gap dipole can be realised without additional correction coils being needed for the clam-shell design. This will allow reducing production cost significantly and paves that path to potentially even make use superconducting coils.

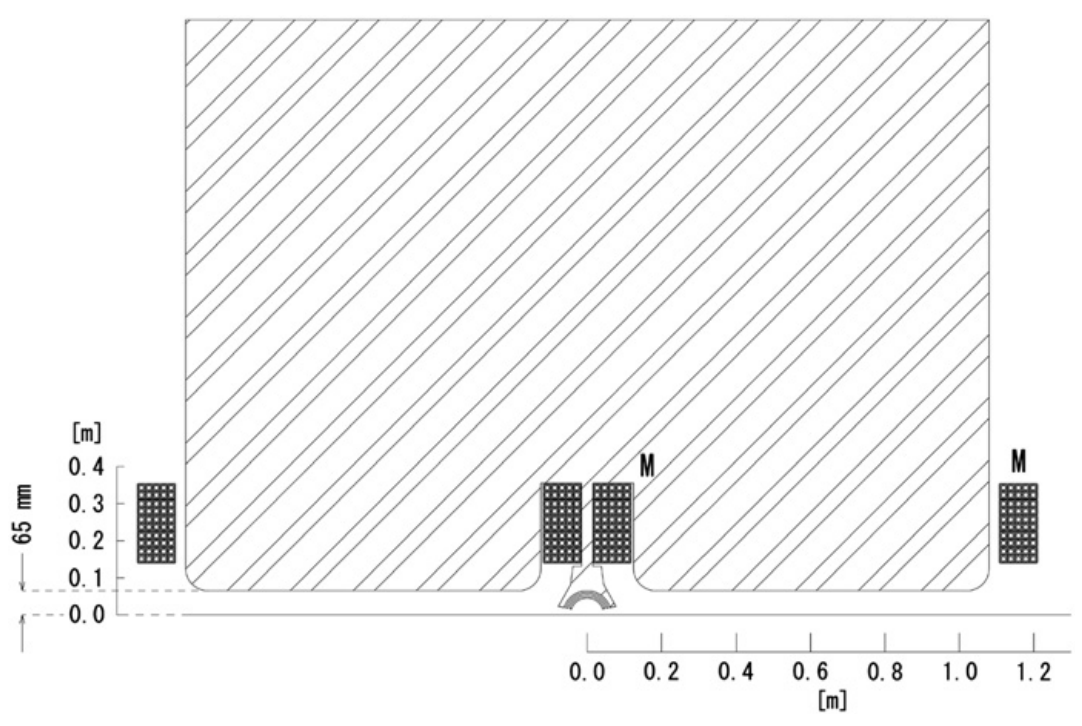

\section{Conclusion \& Further Steps}

The technical design work for the ELISe experiment is brought to a level that allows coming up with technical design reports for its major components, the demanding in-ring electron spectrometer, and the parameters for the bypass section including the interaction zone in short time. Currently, additional efforts are put into the specification of the EAR storage ring, and the electron LINAC shown in Figure 5. The design for the LINAC is based on the positron part of the VEPP-5 injector complex at the BINP in Novosibirsk. 


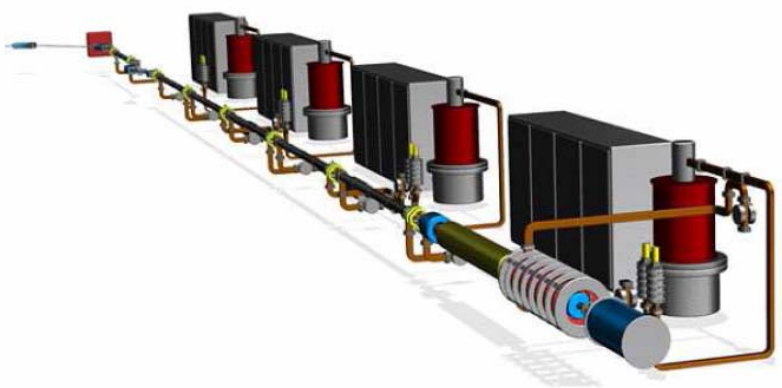

Figure 5:

LINAC for the EAR [9] providing bunches at $10 \mathrm{~Hz}$ repetition rate with a bunch population of a few $10^{10}$ electrons. The electron energy can be tuned from 135-525 MeV.

Additional efforts are to be put into the potential instrumentation of the ELISe experiment. Due to the staging concept for the FAIR facility these developments cannot be carried out without the context of experiments being part of the modularized start version of FAIR. As there is strong overlap between the experimental collaborations, parts of the developments can be pursued already now, as shown for the SOFIA fission experiments, and explored while performing experiments in a different environment. The unrivalled and unique electron scattering program at FAIR remains certainly worthwhile to be carried out, until a solution for building up FAIR's storage ring experiments has eventually been found.

\section{Acknowledgements}

This work was in part supported by the EC via the INTAS programme contract number 05-1000008-8272, by the state of Hesse through the Helmholtz International Center for FAIR within the LOEWE programme, by the Joint Institute for Nuclear Astrophysics (JINA) under NSF grant PHY0822648, by the FAIR-Russia Research Centre (FRRC), and by the Extreme Matter Institute EMMI of the Helmholtzzentrum für Schwerionenforschung, GSI.

\section{References}

[1] FAIR Baseline technical report, Volume 4, Experiment Proposals on Nuclear Structure and Astro Physics (NUSTAR), (2006) via http://www.fair-center.de/Publikationen.171.0.html.

[2] H. Geissel, H. Weick, M. Winkler, G. Münzenberg, V. Chichkine, M. Yavor, et al., Nucl. Inst. and Meth. in Phys. Res. B204, 71 (2003).

[3] T. Suda, M. Wakasugi, et al., Physical Review Letters 102, 102501 (2009).

[4] T. Suda , J. Phys. Conf. Ser. 267, 012008 (2011).

[5] H. Simon, The ELISe experiment at FAIR, Nuclear Physics 787, 102 (2007).

[6] A.N. Antonov et al., Nucl. Inst. and Meth. in Phys. Res. A637, 60 (2011).

[7] G.P.A. Berg et al., Nucl. Inst. and Meth. in Phys. Res. A640, 123 (2011).

[8] T. Adachi et al., Nucl. Inst. and Meth. in Phys. Res. A659, 198 (2011).

[9] P. Shatunov et al., Nucl. Inst. and Meth. in Phys. Res. A, in preparation. 